Proceedings of the Workshop on Automatic Summarization (including DUC 2002), Philadelphia, July 2002, pp. 19-26. Association for Computational Linguistics.

\title{
Using a Text Engineering Framework to Build an Extendable and Portable IE-based Summarisation System
}

\author{
Diana Maynard, Kalina Bontcheva, Horacio Saggion, Hamish Cunningham, Oana Hamza \\ Dept of Computer Science \\ University of Sheffield \\ Sheffield, S1 4DP, UK
}

\begin{abstract}
In this paper we describe how information extraction technology has been used to build a summarisation system in the domain of occupational health and safety. The core of the application is based on named entity recognition using pattern-action semantic grammar rules. Co-occurrence of the named entities is used as a criteria to identify the sentences to be included in the summary. The system is developed and automatically evaluated within the GATE framework, and can easily be extended or ported to new domains.
\end{abstract}

\section{Introduction}

According to (Mani, 2000), the goal of automatic summarization is to take an information source, extract content from it, and present the most important content to the user in a condensed form and in a manner sensitive to the user's or application needs. In this paper we present an IE-based summarisation system (HaSIE), which aims at producing a summary from annual company reports about the companies' performance on Health and Safety issues. The extracted summaries allow the automated production of statistical metrics describing the level of compliance with Health and Safety recommendations and any relevant legislation that may be implemented.

The system detects whether or not the text contains information about health and safety and if so, it extracts relevant passages and conceptual information such as awards, accident rates and number of employees. In this particular application it is vital that we detect correctly whether the report contains health and safety information, because this is one of the main user requirements. The reason why a given report might not contain such information is because the annual company reports contain primarily financial, managerial and performance information.

This summarisation work has been carried out using the GATE architecture. It offers support for the majority of tasks typically performed as part of building a new NLP application, e.g. module development and testing, corpus annotation, and performance evaluation. The framework also has a thorough Unicode support, which enables the development of applications and resources in multiple languages (see (Tablan et al., 2002)).

The rest of the paper is structured as follows. Section 2 describes the information extraction modules used to develop the HaSIE system. Section 3 describes in more detail the process of extracting information and creating the summaries. Next we discuss evaluation in Section 4 and give some preliminary results for our system. Finally, Section 5 discusses how the HaSIE system is related to other approaches to summarisation.

\section{GATE and the HaSIE system}

A set of reusable modules known as ANNIE (A Nearly New Information Extraction system) is 
provided with GATE. These are able to perform basic language processing tasks such as POS tagging and semantic tagging. This eliminates the need for users to keep reinventing the same resources, and provides a good starting point for new applications. We used these components as a basis for building the HaSIE summarisation system described below (see Section 2.2).

\subsection{Finite-state transduction support in GATE}

In order to make it easier to build new processing resources, GATE comes with built-in finitestate transduction capabilities, which we have used as the core of the summarisation process (see Section 3). The transducer runs on grammars written in the JAPE (Java Annotations Pattern Engine) language (Cunningham et al., 2002), which describes patterns to match and annotations to be created as a result.

A JAPE grammar consists of a set of phases, each of which consists of a set of pattern/action rules, and which run sequentially. Patterns can be specified by describing a specific text string, or existing annotations (e.g. annotations created by the tokeniser, gazetteer, part-of-speech tagger, or document format analysis). Rule prioritisation (if activated) prevents multiple assignment of annotations to the same text string.

Creating new modules and applications on the basis of JAPE, such as a summarisation component for another domain, is a low-overhead task, because the user only needs to be concerned with writing new grammar rules (though other modules such as the gazetteer may also need to be updated or modified). The amount of tuning necessary for a new domain can vary depending on the type of information that needs to be extracted, and how similar the domain and text structure is to that which existing components are designed for. For example, we reuse much of the same information about companies, dates, numbers, etc from the default Information Extraction components, so very little tuning was needed in this case, especially since the patterns we aim to identify for health and safety are quite easy to define, but this cannot be guaranteed for all other domains or applications.
So far, we have successfully used JAPE for named entity recognition, sentence splitting, and summarisation, and we intend to experiment with it in other fields such as shallow syntactic parsing. Although at the moment we are using hand-crafted rules, it would be possible for an application to learn rules automatically for the Jape transducers in a manner similar to (Day et al., 1997). These rules could then be verified or amended by a human if necessary, as they are human readable.

\subsection{HaSIE NE Modules}

HaSIE uses a set of Named Entity Recognition modules adapted from the ANNIE information extraction system. HaSIE uses the following processing resources:

- Tokeniser - which splits the text into individual word, number and punctuation tokens.

- Sentence Splitter - which splits the text into individual sentences.

- Part-of-Speech Tagger (Hepple, 2000) which produces a part-of-speech (POS) tag on each token.

- Gazetteer - which contains lists of proper names and keywords used by the grammar.

- Semantic Tagger - a JAPE Transducer which annotates text with information such as entity types.

The first three components are taken directly from ANNIE; the gazetteer and JAPE transducer are modified versions of ANNIE components, which reflect the specific information needs of the project. The HaSIE gazetteer contains additional information about words related specifically to the field of health and safety, such as lists of accident keywords (e.g. "road traffic accident", "fatality", "falls from heights"'), health and safety keywords (e.g. "Lost Workday Cases", "Medical Treatment Cases", "RIDDOR"), and various other lists of more general keywords such as "monitoring", "report", "policy" etc. The HaSIE semantic tagger contains 
hand-coded rules to identify textual patterns which are to be annotated, for example, to find noun phrases which contain information about accidents. These will be described in more detail in the next section.

\section{$3 \quad$ Extracting the relevant information}

The summarisation system was built according to the following steps:

\subsection{Corpus analysis}

A corpus was collected consisting of company reports downloaded from the Web and converted from PDF into HTML. After consultation with experts in the field of health and safety as to which types of information were relevant, the reports were analysed manually and guidelines were drawn up about relevant sentences and phrases to be extracted. These were then analysed further by the system developers (computational linguists) in order to establish which entities should be recognised, and how the relevant material could be extracted. This list included key incident and accident words and phrases, health and safety-related words and phrases, names of organizations, job titles, locations, percentages, figures, and dates.

\subsection{Extraction of entities}

The entity extraction was performed by adapting the gazetteer and semantic tagger modules from ANNIE detailed in Section 2.2. The gazetteer lists were updated with keywords, and new rules were added to the semantic tagger. These modules annotate specific words and phrases related to health and safety (e.g. "HSE", "Occupational Health") with an HSE label, accidents (e.g. "accident", "injury", "death") with an Accident label, and dates, company names, etc. with similar relevant labels. Some of these annotations, such as Company Name, are output directly (see Section 3.4 ); others are used in the sentence generation phase (see Section 3.3). Figure 1 shows part of a sample text (the Debenhams company report) annotated with such entities.
The annotation types extracted in this phase are: Company, Organization, Jobtitle ${ }^{1}$, Accident, HSE, Date, Percent, Money and Number. With the exception of Company, Accident and HSE, these types (and the rules used to extract them) are the same as those used in the default ANNIE system. The difference between Company and Organization is that Company depicts the name of the company which has produced the report, while Organization depicts any organisation mentioned in the document (which may or may not be a company name).

The following rule shows how we extract an HSE annotation, using the result of gazetteer lookup and part-of-speech tagging. We look for a Jobtitle annotation (found in a previous phase of the grammar), followed by one or more numbers, common nouns or adjectives (in any combination) followed by something which has been found in a gazetteer list of HSE keywords. (The POS tag is specified by the feature "category" on the token.) If this pattern is matched, then the whole pattern is annotated as an HSE.

Rule: HSE2

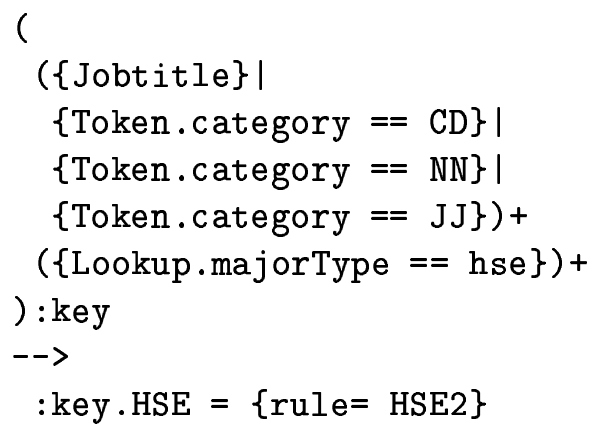

\subsection{Extracting sentences}

The next step uses another JAPE transducer to define rules which extract sentences or paragraphs which describe health and safety information. The transducer depends on previous annotations such as the result of sentence splitting, tokenisation, and the initial annotations produced in the entity extraction phase. We extract sentences describing specific information, such as accident and incident rates, and also

\footnotetext{
${ }^{1}$ We identify this because we want to know whether there is somebody in the company whose role is to look after health and safety matters.
} 


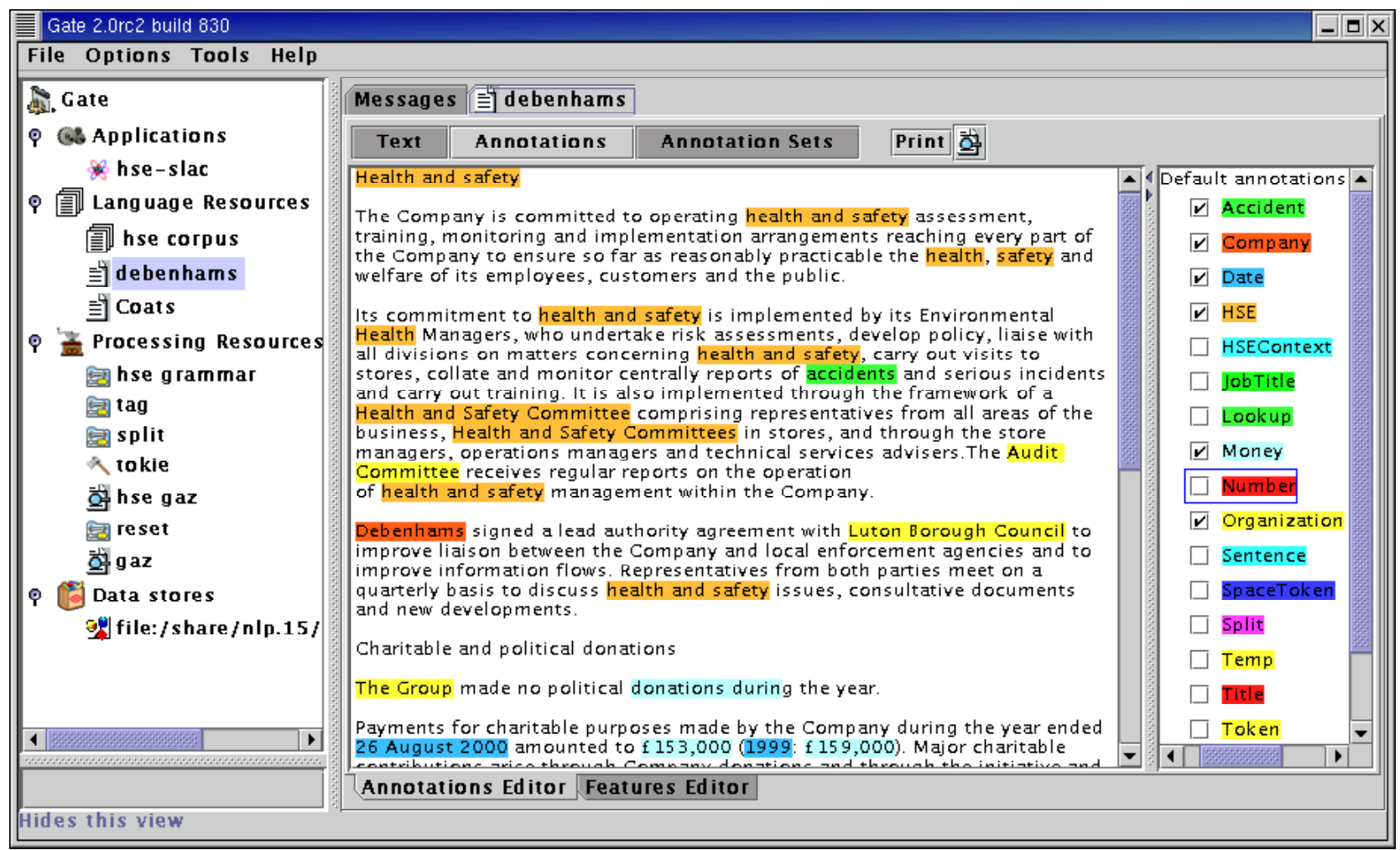

Figure 1: Debenhams report annotated with entities

paragraphs which describe more general issues about health and safety. The reason we extract whole paragraphs is that there is often relevant information in the whole paragraph which would be missed if we only extracted the sentences relating specifically to health and safety.

\subsubsection{Paragraph Annotations}

We explain below 3 typical rules used in the generation of HSE paragraphs.

The first rule tries to match paragraphs containing an HSE entity annotation, followed by one or more Accident annotations, Environment annotations, or HSE Keys (possibly separated by other words). An HSE Key is a word which in itself does not represent something related to health and safety, but when combined with an HSE annotation suggests more strongly the presence of something related to health and safety. For example "report" on its own does not provide conclusive evidence, but when found with "health" provides a much stronger clue. "Environment" annotations on their own again do not provide much evidence, but when combined with an HSE annotation are again much more strongly linked with health and safety. If the rule finds a successful match, the whole paragraph is annotated.

The second rule tries to match paragraphs containing an HSE annotation, followed by one or more other HSE annotations or Organization annotations (possibly separated by other words). An HSE annotation refers to a word of phrase directly related to health and safety, an Organization annotation refers to a company name or name of another organization such as "Health and Safety Executive" or "EC". If the rule finds a successful match, the whole paragraph is annotated.

The third rule looks for an HSE Key followed by an HSE annotation (possibly separated by other words). If the rule finds a successful match, the whole paragraph is annotated. Since this is quite a general rule, it only gets fired if both the other rules fail to match. 


\subsubsection{Sentence Annotations}

We also identify sentences about more specific facts such as accidents and illnesses, e.g.

" The accident frequency ratio for construction projects was $0.4(0.49)$ per 100,000 hours worked, less than a third of the national accident frequency rate in the construction sector.'

These are identified using rules based on entities such as percentages, accident entities, and numbers.

We currently use two rules to identify accident statistics. The first rule tries to match a Number annotation (written in figures) followed immediately by an Accident annotation. The second rule is more complicated, and tries to match an Accident annotation, followed immediately by one or more Number annotations (in figures) or Percentage annotations, followed optionally by further Accident, number or percentage annotations. Number and Percentage annotations are produced during earlier phases of the grammar, and are based largely on gazetteer lookup and string identification (e.g. looking for the "\%" sign). If this pattern is matched, the whole sentence is again identified.

\subsection{Populating a database}

Finally, the relevant annotated parts of the document are output by the system in a comma separated file format, which is then imported in an Access database. The users inspect the summarisation results using a form interface (see Figure 2) and also SQL queries (e.g., to find out how many companies do not discuss health and safety issues in their annual reports). Prior to using the IE-based summarisation system, such analysis was performed manually, which involved locating the necessary information in several hundred reports, each between 50 and 100 pages long.

\section{Evaluation}

Evaluations of text summarization systems can be intrinsic or extrinsic (Sparck Jones and Gal- liers, 1995): intrinsic evaluation measures the content of the summary by a comparison with an "ideal" or "target" summary. Extrinsic evaluation measures how helpful summaries are in the completion of a given task, for example in question answering or text categorization. When the evaluation is done by comparing extracted textual units (sentences or paragraphs) to a set of ideal textual units, then co-selection is measured by precision, recall and F-score (Firmin and Chrzanowski, 1999). While many researchers have resisted these measures because in generic summarization it is recognized that there is no "ideal summary" (Jing et al., 1998), in our domain-dependent IE-based summarization, there is a clear idea of what information should be included in the summary and which articles deal with health and safety information.

First, we carried out formative evaluation, involving input from our users, as part of the system development cycle. We processed 36 company reports (8.9 MB of HTML text) with HaSIE and asked one of our users to mark up in the resulting summaries $(97.6 \mathrm{~KB})$ the sentences which they found highly relevant, moderately relevant, and completely irrelevant.

In an example taken from the Burmah Castrol company report, the following section was marked as highly relevant:

Burmah Castrol is committed to effective occupational health practices and procedures. The larger sites in the businesses have their own arrangements for monitoring their health and safety performance and, where appropriate, health surveillance.

The next section was marked as moderately relevant:

Underpinning the reduction of environmental impacts has been the introduction of training programmes to raise awareness of environmental issues. Two international Safety Health and Environment (SHE) meetings were held in 1999 involving SHE specialists, business managers, marketers and customers.

Finally, this section was marked as irrelevant: 


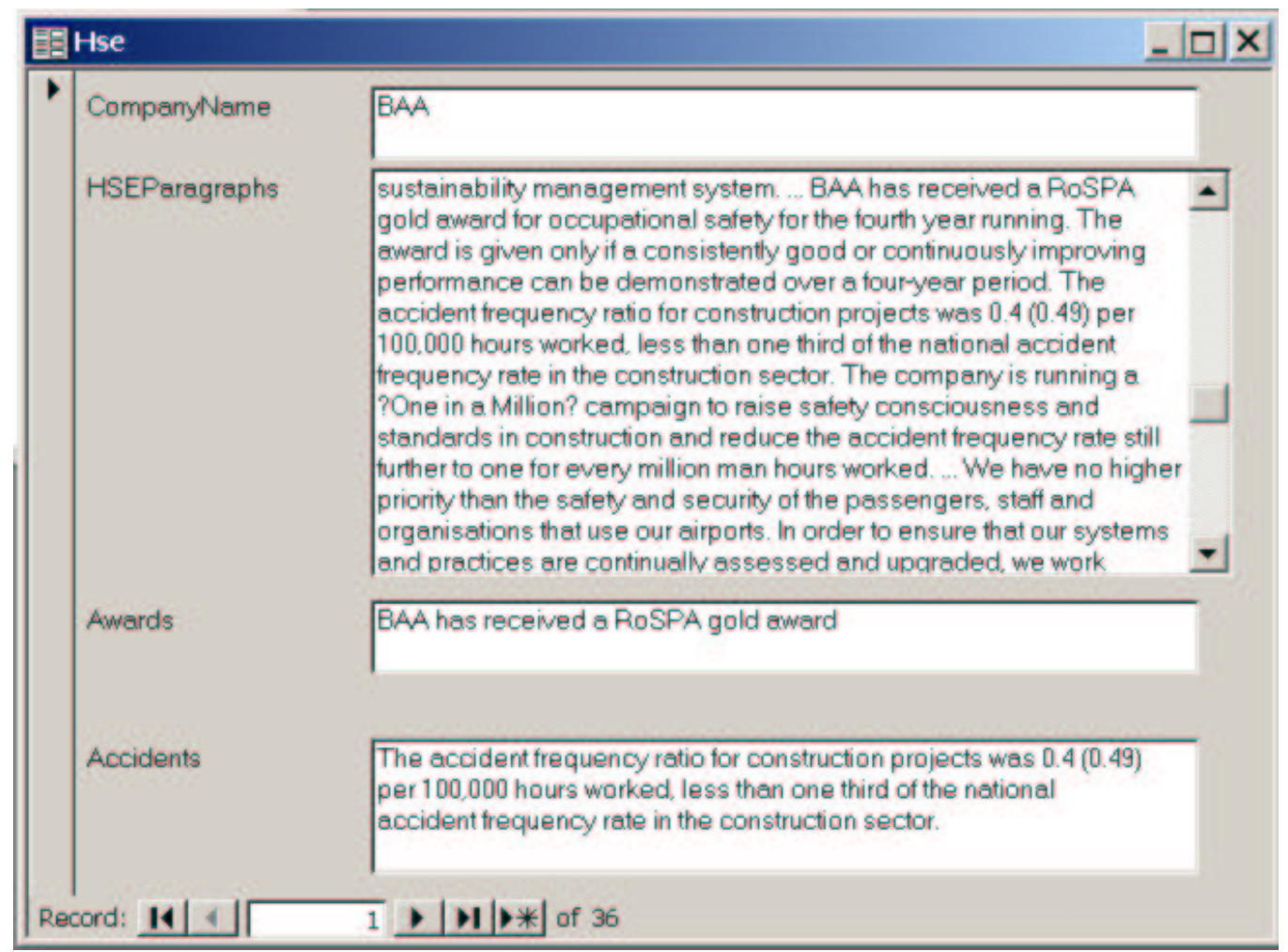

Figure 2: The Access form used for report summary analysis

Working with the charity "Riders for Health', our contribuition to a school for excellence in Zimbabwe will provide outreach health workers with training in motorcycle riding and maintenance. This will help maintain vital services to rural areas.

The principle behind this was that the users considered it top priority for the system to find the highly relevant sections; ideally, the system should also find the moderately relevant sections, but this was not so important; the system should not identify any of the irrelevant sections, although this was again less important than finding the relevant sections. This meant that the system should be tuned slightly more towards recall than Precision. To avoid high recall errors, however, the system deliberately identified some non-relevant sections (in order to ensure that they are not annotated as relevant).

\subsection{Evaluation tools}

A vital part of any language processing application is the evaluation of its performance, and a development environment for this purpose would not be complete without some mechanisms for its measurement in a large number of test cases. GATE contains two such mechanisms: an evaluation tool (AnnotationDiff) which enables automated performance measurement and visualisation of the results, and a benchmarking tool, which enables the tracking of a system's progress and regression testing.

Gate's AnnotationDiff tool enables two sets of annotations on a document to be compared, in order to either compare a system-annotated text with a reference (hand-annotated) text, or to compare the output of two different versions of the system (or two different systems). For each annotation type, figures are generated for precision, recall, F-measure and false positives.

The AnnotationDiff viewer displays the two sets of annotations, marked with different colours (similar to 'visual diff' implementations 
such as in the MKS Toolkit or TkDiff). Annotations in the key set have two possible colours depending on their state: white for annotations which have a compatible (or partially compatible) annotation in the response set, and orange for annotations which are missing in the response set. Annotations in the response set have three possible colours: green if they are compatible with the key annotation, blue if they are partially compatible, and red if they are spurious. In the viewer, two annotations will be positioned on the same row if they are co-extensive, and on different rows if not.

GATE's benchmarking tool differs from the AnnotationDiff in that it enables evaluation to be carried out over a whole corpus rather than a single document. It also enables tracking of the system's performance over time. Furthermore, the system can be run in verbose mode, where for each performance figure below a certain threshold (set by the user), the noncoextensive annotations (and their corresponding text) will be displayed. This information is useful e.g., when developing new JAPE grammar rules to cover cases currently missed by the system.

\subsection{Results}

Preliminary results showed that the system had identified correctly that 8 of the reports did not contain relevant information. From the remaining 28 summaries, only 10 had some irrelevant sentences, but these sentences never constituted more than $50 \%$ of the summary. These results led to changing HaSIE to account better for ambiguous keywords, such as "health", because often such words occur in irrelevant terms like "health care" and "health insurance". By recognising such terms as spurious, we prevented them from leading to the (erroneous) recognition of sentences containing them as relevant. After these changes were implemented, 7 of the 10 problematic summaries no longer contained the irrelevant senteces.

After improving the system to deal with keyword ambiguities, we then created a manually annotated HSE summary corpus for 80 of the company reports. To this end, we used HaSIE to bootstrap the annotation process and then correct its results manually, using the visual document annotation editor from GATE. This corpus was used to measure the system's performance, using GATE's evaluation tools described in Section 4.1.

Results show that overall the system achieved $78 \%$ precision and $80 \%$ recall, which is consistent with the performance estimates we obtained from the formative evaluation, after we discarded the irrelevant sentences which were no longer included after the ambiguity improvements. Furthermore, in $70 \%$ of the reports, the system identified the presence of health and safety data correctly, and in $15 \%$ of these, the system found this data correctly where humans had failed to find it. The system only missed this data in 1 report, and in all the remaining reports, the system correctly identified that there was no health and safety data present. The system generated 70 pages output from approximately 6000 pages of reports, thereby creating a substantial reduction in the workload of the experts who wished to make use of the relevant data.

Although 80 reports might seem a small number of texts on which to perform an evaluation, this is the current scale at which human analysts are performing this task manually each year. The creation of a reliable automatic system will allow the process to scale up into the thousands and ultimately be able to analyse the HSE data in all reports of public UK companies.

\section{Related Work}

Unlike other knowledge-based approaches to summarization that depend on rich conceptual structures (Hahn, 1990; Rau et al., 1989), we rely on domain-specific terminology and robust Information Extraction techniques. Closely related to our approach is Concept Based Abstracting (CBA) (Paice and Oakes, 1999) where shallow processing is used to instantiate a semantic frame containing the most important concepts of the source document. Unlike CBA, which produces a short textual abstract, our system produces a set of text passages and instan- 
tiates a number of slots (e.g. company, number of employees, etc.) which are used by an information analyst to produce health and safety reports. Our rules for passage extraction rely on concept co-occurrence, and so are similar to the contextual templates employed in other IE approaches to summarization (Paice and Jones, 1993; Saggion and Lapalme, 2000).

\section{Conclusion and Future Work}

In this paper we have presented how GATE was used as a development environment for a summarization system. Our focus was on the adaptation of general natural language engineering tools to an specific summarization problem. While we have relied completely on IE for the purpose of this application, GATE also allows the programmer to easily deploy methods within the framework for computing surface level indicators of salience. In fact, several such GATEbased modules (e.g., sentence position, $t f * i d f$ ) are currently being developed by one of the authors.

\section{References}

H. Cunningham, D. Maynard, K. Bontcheva, V. Tablan, and C. Ursu. 2002. The GATE User Guide. http://gate.ac.uk/.

D. Day, J. Aberdeen, L. Hirschman, R. Kozierok, P. Robinson, and M. Vilain. 1997. MixedInitiative Development of Language Processing Systems. In Proceedings of the 5th Conference on Applied Natural Language Processing (ANLP-97).

T. Firmin and M.J. Chrzanowski. 1999. An Evaluation of Automatic Text Summarization Systems. In I. Mani and M.T. Maybury, editors, Advances in Automatic Text Summarization, pages 325-336.

Udo Hahn. 1990. Topic Parsing: Accounting for Text Macro Structures in Full-Text Analysis. Information Processing \& Management, 26(1):135170.

Mark Hepple. 2000. Independence and commitment: Assumptions for rapid training and execution of rule-based POS taggers. In Proceedings of the 38th Annual Meeting of the Association for Computational Linguistics (ACL-2000), Hong Kong, October.
Hongyan Jing, Kathleen McKeown, Regina Barzilay, and Michael Elhadad. 1998. Summarization Evaluation Methods: Experiments and Analysis. In Intelligent Text Summarization. Papers from the 1998 AAAI Spring Symposium. Technical Report SS-98-06, pages 60-68, Standford (CA), USA, March 23-25. The AAAI Press.

Inderjeet Mani. 2000. Automatic Text Summarization. John Benjamins Publishing Company.

Chris D. Paice and Paul A. Jones. 1993. The Identification of Important Concepts in Highly Structured Technical Papers. In R. Korfhage, E. Rasmussen, and P. Willett, editors, Proc. of the 16th ACM-SIGIR Conference, pages 69-78.

Chris D. Paice and Michael P. Oakes. 1999. A Concept-Based Method for Automatic Abstracting. Technical Report Research Report 27, Library and Information Commission.

Lisa F. Rau, Paul S. Jacobs, and Uri Zernik. 1989. Information Extraction and Text Summarization using Linguistic Knowledge Acquisition. Information Processing \& Management, 25(4):419-428.

H. Saggion and G. Lapalme. 2000. Concept Identification and Presentation in the Context of Technical Text Summarization. In Proceedings of the Workshop on Automatic Summarization. ANLPNAACL2000, Seattle, WA, USA, 30 April. Association for Computational Linguistics.

K. Sparck Jones and J.R. Galliers. 1995. Evaluating Natural Language Processing Systems: An Analysis and Review. Number 1083 in Lecture Notes in Artificial Intelligence. Springer.

V. Tablan, C. Ursu, K. Bontcheva, H. Cunningham, D. Maynard, O. Hamza, Tony McEnery, Paul Baker, and Mark Leisher. 2002. A unicode-based environment for creation and use of language resources. In Proceedings of 3rd Language Resources and Evaluation Conference. forthcoming. 